\title{
Normal values of modified functional reach test in Indian school going children of age 6 to 12 years
}

\author{
Abhijeet Arun Deshmukh ${ }^{1}$, Mrunmayee Mukund Joshi2 ${ }^{2, *}$ \\ ${ }^{\mathbf{1}}$ Assistant Professor, ${ }^{2}$ Internee, Dept. of Neuro-Physiotherapy, VSPM's College of Physiotherapy, Nagpur, Maharashtra, India
}

*Corresponding Author:

Email: mrunmayeemjoshi20@gmail.com

\begin{abstract}
Modified functional reach test is valid and reliable tool to assess sitting balance in children in forward and lateral direction. Normative data of modified functional reach test for school going children has not been established yet in central India, as well correlation of anthropometric data with reach distance values not studied, hence this study was undertaken. Total 280 children (6 to 12 years), 140 Boys and 140 Girls, were selected by stratified random sampling method, subdivided into 7 subgroups (10B, 10G in each group) from two schools. Height was measured using stature meter, trunk length and leg length were measured using measuring tape, weight was measured using weighing machine, spinal flexibility was tested with the help of sit and reach test, hamstring angle was measured using goniometer by 90-90 test. Child reached with dominant shoulder at 90 degree of flexion and abduction in forward and lateral direction respectively while sitting on height adjustable stool with hip and knee at 90 degrees and feet placed neutral on ground. The reach distance was measured using a yard stick mounted on the wall, at the height of child's shoulder. The mean of three successive trials was calculated. Karl Pearson correlation moment product was used to determine correlation between age, gender and anthropometric measurements with modified forward and lateral reach. Normal values of modified forward and lateral reach were observed as 19.53 to $26.48 \mathrm{~cm}$ and 17.68 to $22.50 \mathrm{~cm}$ respectively. Height and weight correlated significantly with modified functional reach values.
\end{abstract}

Keywords: Sitting balance assessment, School children, Modified functional reach, Modified lateral reach, Height, Weight.

\section{Introduction}

Balance is the condition in which all the forces acting on the body are balanced so that the centre of mass (COM) is within the limits of stability and the boundaries of base of support (BOS). ${ }^{1}$ The ability to maintain a posture such as balancing in a standing or sitting position, is operationally defined as static balance. ${ }^{1}$ The ability to maintain postural control during movements, such as reaching for an object or walking across objects, is operationally termed as dynamic balance ${ }^{1}$. Balance emerges from a complex interaction of sensory systems (afferent-visual, cutaneous, proprioceptive), motor systems (effector- muscles, bones, joints), vestibular system and central nervous system (CNS). ${ }^{1}$ These systems work in a coordinated way in order to maintain static postures and during dynamic tasks. ${ }^{1}$ These systems independently predominate at various rates as age advances. Infants and young children (aged 4 months-2 years) are dependent on the visual system to maintain balance. At 3-6 years of age, children begin to use somatosensory information appropriately. ${ }^{1}$ At the age of 6-8 years, a transition occurs in organisation of postural behaviour by maturation of somatosensory and vestibular system ${ }^{2-4}$ and beyond this age, the effect of vision becomes less dominant when compared with other systems. ${ }^{5}$ Finally, at 7-10 years of age, children are able to resolve a sensory conflict and appropriately utilize the vestibular system as a reference along with other systems. ${ }^{1}$

Sitting is a milestone achieved by a typically developing child as a part of normal motor development, at the age of 5-8 months and reaching activity is also achieved at the same age which forms the basis for the dynamic balance in sitting position. ${ }^{6}$ The ability to balance while reaching for a variety of objects both within and beyond arm's length is important for daily activities. ${ }^{6}$ In children, sitting balance plays an important role during functional activities such as maintaining or attaining sitting posture, dressing, transferring, bathing, toileting and eating activities and also in activities like playing, hobby, recreation, schooling etc. ${ }^{2,7}$

In individuals who have neuromuscular dysfunction or have abnormal motor development may show variation in postural adjustments leading to impaired balance. ${ }^{8-12}$ To assess balance, various age specific scales, tests and measures are available that assess sitting balance individually or as a part of the test such as paediatric clinical test of sensory interaction for balance, Seated postural control measure, Level of sitting ability scale, Paediatric balance scale, Bruininks-Oseretsky test of motor proficiency, Timed get up and go test, Sitting Assessment for children with neuromotor dysfunction, Gross motor function measure, Chailey levels of ability, (mFRT), etc. ${ }^{10,13-18}$

Modified functional reach test used for balance evaluation is less time consuming and requires no equipment, easily understood by all age groups and hence is widely used as objective measure to assess dynamic sitting balance. ${ }^{6,10,19}$ It is defined as the maximal distance one can reach beyond arm length while maintaining a fixed base of support in sitting. Modified functional reach test examines movements in two directions, forward and lateral in sitting. It is having high validity and reliability among both adult and paediatric population. ${ }^{10,19,20}$ The construct validity of mFRT is supported in children typically developing and in children with cerebral palsy. ${ }^{19}$ The modified functional reach test showed intra class coefficient (ICC), intrarater, and inter-rater reliability as $0.84,0.71$, and 0.71 
respectively. ${ }^{19}$ There are several factors affecting balance like age, height, weight and other anthropometric measurements $\mathrm{s}^{3,10,21,22}$ which show vast difference in balance scores among children in developing ages. Till date, normal reference values for $\mathrm{mFRT}$ are not available for paediatric population between 6-12 years of age in India. Hence, the primary purpose of the study was to establish clinical reference values for Indian school going children.

\section{Materials and Methods}

Total 280 children were selected of both genders (140 boys and 140Girls) by multistage stratified sampling method. A cross sectional study was performed on normal healthy children of age group 6-12 years selected from one urban school and one rural school of Nagpur. Children were divided into 7 subgroups depending upon age i.e. 6-12 years. The study was carried out over a period of 3 months. Children having any neuromuscular or musculoskeletal disorders $^{6}$, severe known visual defects, ${ }^{6}$ history of ear infection/pain since last 6 months were excluded from the study.

Anthropometric measurements of height and weight were done by using stature meter and weighing machine respectively. BMI was calculated and children between the range of 13.37 to $22.15 \mathrm{~kg} / \mathrm{m}^{2}$ i.e. $\left(10^{\text {th }}\right.$ and $90^{\text {th }}$ percentile for age 6-12 years.) were included. Trunk length and leg length were measured using measuring tape. ${ }^{6,23}$ Universal half Goniometer was used to measure Hamstring angle ${ }^{24}$ and Sit and Reach apparatus was used to assess trunk flexibility. ${ }^{6}$ Children showing no tightness in $90-90$ hamstring tightness test were included.

A stool with adjustable height was arranged near the wall, where child seated independently with back straight and feet pelvis width apart, touching the floor completely. A yard stick was arranged at the height of the shoulder of the child. Forward reach and lateral reach of the children were measured by asking the child to bend with his/her arm flexed at 90 degrees for forward reach and 90 degrees abduction for lateral reach $^{6}$ (Fig. 1-4). Three successive measurements were taken in centimetres. Mean of the three finding was calculated for each child. No unexpected responses or injuries occurred during testing.

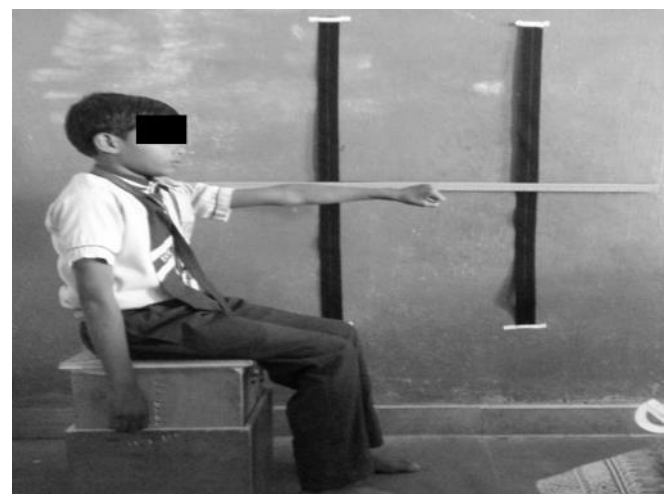

Fig. 1: Starting position for modified forward reach test

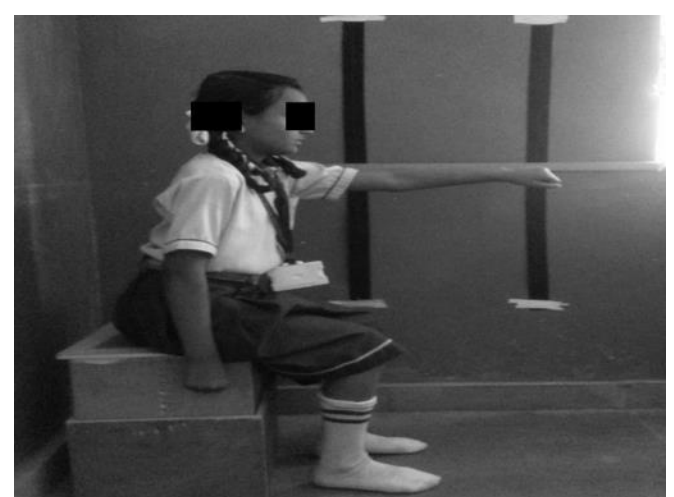

Fig. 2: End position for modified forward reach test

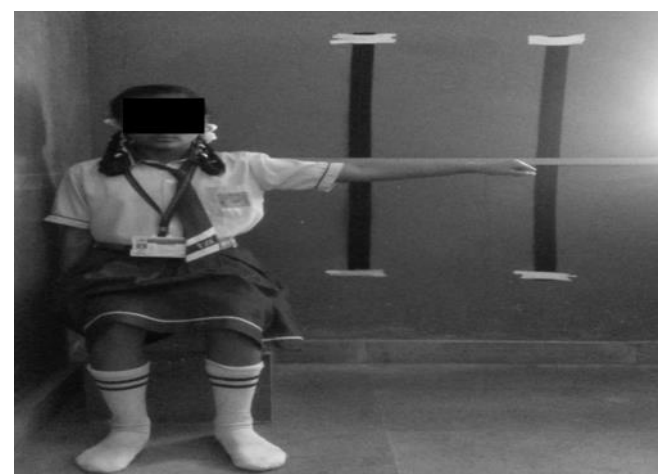

Fig. 3: Starting position for modified lateral reach test

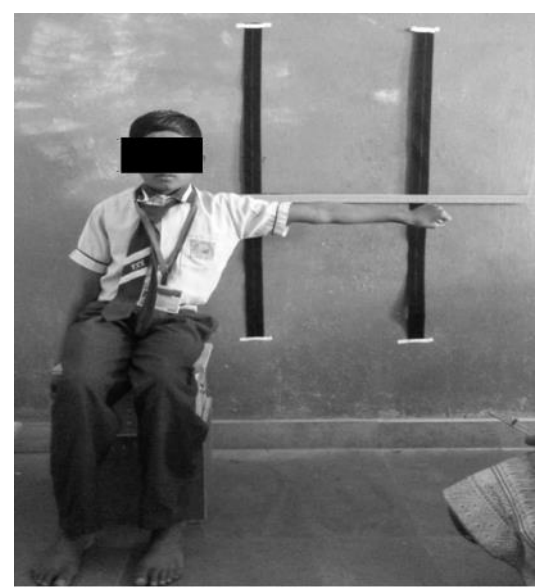

Fig. 4: End position modified lateral reach test

\section{Data Analysis}

SPSS version 20.0 was used for statistical analysis. Student's t-test was used to obtain normal values of modified functional reach test. Karl Pearson correlation moment product was used to determine correlation between age, gender, height, weight, trunk length, leg length, sit and reach distance and hamstring angle with mFRT and mLRT. Statistics were determined at $95 \%$ confidence interval.

\section{Results}

The mean and Standard deviation of anthropometric measurements, sit and reach test distance and 90-90 hamstring tightness angle were explained in Table 1 . Normal values of mFRT and mLRT ranged from19.53 \pm 
$3.57 \mathrm{~cm}$ to $26.48 \pm 5.27 \mathrm{~cm}$ and $17.68 \mathrm{~cm} \pm 2.59 \mathrm{~cm}$ to 22.50 $\mathrm{cm} \pm 3.75 \mathrm{~cm}$ among both genders between the ages of 6-12 years respectively. (Table 2 )

Correlation of mFRT and mLRT with anthropometric measures, trunk flexibility and hamstring angle (Table 3, 4):

The values of mFRT and mLRT increases with age in both genders and showed a highly significant correlation with height, leg length, trunk length. The mFRT distances showed a significant correlation with weight in both genders whereas, mLRT distances were correlated significantly with weight in girls, but not in boys. The mFRT distances showed a significant correlation with sit and reach test distance (trunk flexibility) in boys but not in girls. The
mLRT distances did not show correlation with trunk flexibility in both genders. Both distances did not show significant correlation with BMI and hamstring angle in both genders.

For children of age 6-12 years mLR mean distance value could be predicted significantly (Table 5) $\left(\mathrm{R}^{2}=0.48\right)$ by $\mathrm{mFR}$ mean distance and vice versa in both genders by using following formula:

$\mathrm{mLR}(\mathrm{cm})=7.28+[0.607 \times \mathrm{mFR}(\mathrm{cm})]$

On evaluation of lateral reach test, it was found that there was no significant difference between right and the left side reach values (Graph 1).

Table 1: Mean and standard deviation of demographic data, sit and reach distance, hamstring angle

\begin{tabular}{|c|c|c|c|c|c|c|c|c|}
\hline Age & Gender & $\begin{array}{c}\text { Height } \pm \text { SD } \\
(\mathrm{cm})\end{array}$ & $\begin{array}{c}\text { Weight } \pm \text { SD } \\
(\mathrm{kg})\end{array}$ & $\begin{array}{c}\text { BMI } \pm \\
\text { SD }\left(k g / m^{2}\right)\end{array}$ & $\begin{array}{c}\text { Leg } \\
\text { Length } \pm \text { SD } \\
(\mathrm{cm})\end{array}$ & $\begin{array}{c}\text { Trunk } \\
\text { Length } \pm \text { SD } \\
(\mathrm{cm})\end{array}$ & $\begin{array}{c}\text { Sit and reach } \\
\text { distance } \pm \\
\mathrm{SD}(\mathrm{cm})\end{array}$ & $\begin{array}{c}\text { Hamstring angle } \pm \\
\text { SD(degrees) }\end{array}$ \\
\hline \multirow[t]{2}{*}{6} & G & $\begin{array}{c}113.35 \pm \\
3.67\end{array}$ & $\begin{array}{c}20.25 \pm \\
3.66\end{array}$ & $\begin{array}{c}15.70 \pm \\
2.27\end{array}$ & $\begin{array}{c}33.90 \pm \\
1.48\end{array}$ & $\begin{array}{c}44.65 \pm \\
1.53\end{array}$ & $\begin{array}{c}8.50 \pm \\
3.87\end{array}$ & $\begin{array}{c}173.50 \pm \\
10.52\end{array}$ \\
\hline & B & $\begin{array}{c}114.70 \pm \\
5.13\end{array}$ & $\begin{array}{c}18.80 \pm \\
2.84\end{array}$ & $\begin{array}{c}14.15 \pm \\
1.38\end{array}$ & $\begin{array}{c}34.00 \pm \\
1.94\end{array}$ & $\begin{array}{c}43.20 \pm \\
2.73\end{array}$ & $\begin{array}{c}8.35 \pm \\
4.42\end{array}$ & $\begin{array}{c}172.50 \pm \\
8.95\end{array}$ \\
\hline \multirow[t]{2}{*}{7} & $\mathrm{G}$ & $\begin{array}{c}117.60 \pm \\
4.81\end{array}$ & $\begin{array}{c}20.85 \pm \\
3.36\end{array}$ & $\begin{array}{c}14.80 \pm \\
1.90\end{array}$ & $\begin{array}{c}35.55 \pm \\
2.13\end{array}$ & $\begin{array}{c}45.60 \pm \\
2.72\end{array}$ & $\begin{array}{c}9.15 \pm \\
3.20\end{array}$ & $\begin{array}{c}174.75 \pm \\
9.79\end{array}$ \\
\hline & $\mathrm{B}$ & $\begin{array}{c}122.10 \pm \\
5.23\end{array}$ & $\begin{array}{c}22.90 \pm \\
5.27\end{array}$ & $\begin{array}{c}15.30 \pm \\
2.47\end{array}$ & $\begin{array}{c}37.00 \pm \\
2.31\end{array}$ & $\begin{array}{c}47.15 \pm \\
1.69\end{array}$ & $\begin{array}{c}7.05 \pm \\
2.80\end{array}$ & $\begin{array}{c}160.75 \pm \\
9.49\end{array}$ \\
\hline \multirow[t]{2}{*}{8} & $\mathrm{G}$ & $\begin{array}{c}125.85 \pm \\
4.74\end{array}$ & $\begin{array}{c}23.10 \pm \\
2.78\end{array}$ & $\begin{array}{c}14.45 \pm \\
1.19\end{array}$ & $\begin{array}{c}39.55 \pm \\
2.41\end{array}$ & $\begin{array}{c}47.80 \pm \\
2.84\end{array}$ & $\begin{array}{c}10.30 \pm \\
1.49\end{array}$ & $\begin{array}{c}167.25 \pm \\
6.58\end{array}$ \\
\hline & $\mathrm{B}$ & $\begin{array}{c}126.55 \pm \\
6.37\end{array}$ & $\begin{array}{c}23.30 \pm \\
5.14\end{array}$ & $\begin{array}{c}14.45 \pm \\
2.43\end{array}$ & $\begin{array}{c}38.35 \pm \\
2.88\end{array}$ & $\begin{array}{c}46.10 \pm \\
3.09\end{array}$ & $\begin{array}{c}10.40 \pm \\
1.78\end{array}$ & $\begin{array}{c}169.50 \pm \\
7.76\end{array}$ \\
\hline \multirow[t]{2}{*}{9} & $\mathrm{G}$ & $\begin{array}{c}131.55 \pm \\
6.24 \\
\end{array}$ & $\begin{array}{c}28.55 \pm \\
5.83 \\
\end{array}$ & $\begin{array}{c}16.40 \pm \\
2.37 \\
\end{array}$ & $\begin{array}{c}41.65 \pm \\
2.81 \\
\end{array}$ & $\begin{array}{c}49.15 \pm \\
3.81 \\
\end{array}$ & $\begin{array}{c}13.50 \pm \\
3.95 \\
\end{array}$ & $\begin{array}{l}173 \pm \\
11.16 \\
\end{array}$ \\
\hline & B & $\begin{array}{c}131.05 \pm \\
4.72 \\
\end{array}$ & $\begin{array}{c}27.50 \pm \\
7.41 \\
\end{array}$ & $\begin{array}{c}15.85 \pm \\
3.78 \\
\end{array}$ & $\begin{array}{c}40.65 \pm \\
2.18 \\
\end{array}$ & $\begin{array}{c}49.75 \pm \\
3.62 \\
\end{array}$ & $\begin{array}{c}13.30 \pm \\
4.24 \\
\end{array}$ & $\begin{array}{c}169.75 \pm \\
13.52 \\
\end{array}$ \\
\hline \multirow[t]{2}{*}{10} & $\mathrm{G}$ & $\begin{array}{c}133.10 \pm \\
6.46\end{array}$ & $\begin{array}{c}29.65 \pm \\
6.23 \\
\end{array}$ & $\begin{array}{c}16.55 \pm \\
2.54\end{array}$ & $\begin{array}{c}42.70 \pm \\
2.69 \\
\end{array}$ & $\begin{array}{c}50.85 \pm \\
3.31 \\
\end{array}$ & $\begin{array}{c}11.95 \pm \\
3.83 \\
\end{array}$ & $\begin{array}{c}171.75 \pm \\
8.47\end{array}$ \\
\hline & B & $137.75 \pm 8.14$ & $\begin{array}{c}30.75 \pm \\
6.23\end{array}$ & $\begin{array}{c}16.10 \pm \\
2.55\end{array}$ & $\begin{array}{c}42.90 \pm \\
2.78\end{array}$ & $\begin{array}{c}50.85 \pm \\
3.04\end{array}$ & $\begin{array}{c}10.05 \pm \\
3.90\end{array}$ & $\begin{array}{c}169.25 \pm \\
10.16\end{array}$ \\
\hline \multirow[t]{2}{*}{11} & $\mathrm{G}$ & $\begin{array}{c}144.70 \pm \\
9.30\end{array}$ & $\begin{array}{c}33.80 \pm \\
8.19\end{array}$ & $\begin{array}{c}15.95 \pm \\
2.25 \\
\end{array}$ & $\begin{array}{c}49.80 \pm \\
5.68 \\
\end{array}$ & $\begin{array}{c}50.10 \pm \\
6.96\end{array}$ & $\begin{array}{c}12.55 \pm \\
4.22 \\
\end{array}$ & $\begin{array}{c}164.75 \pm \\
11.97\end{array}$ \\
\hline & B & $141.00 \pm 6.03$ & $\begin{array}{c}37.90 \pm \\
11.48\end{array}$ & $\begin{array}{c}19.00 \pm \\
5.70\end{array}$ & $\begin{array}{c}48.90 \pm \\
4.29\end{array}$ & $\begin{array}{c}49.75 \pm \\
5.86\end{array}$ & $\begin{array}{c}12.95 \pm \\
3.45\end{array}$ & $\begin{array}{c}168.75 \pm \\
12.76\end{array}$ \\
\hline \multirow[t]{2}{*}{12} & G & $\begin{array}{c}150.55 \pm \\
7.14\end{array}$ & $\begin{array}{c}40.50 \pm \\
9.86\end{array}$ & $\begin{array}{c}17.70 \pm \\
3.04\end{array}$ & $\begin{array}{c}47.30 \pm \\
2.77\end{array}$ & $\begin{array}{c}58.50 \pm \\
5.35\end{array}$ & $\begin{array}{c}9.50 \pm \\
2.41\end{array}$ & $\begin{array}{c}166.25 \pm \\
9.85\end{array}$ \\
\hline & $\mathrm{B}$ & $\begin{array}{c}149.30 \pm \\
9.29\end{array}$ & $\begin{array}{c}39.80 \pm \\
9.23\end{array}$ & $\begin{array}{c}17.65 \pm \\
3.10\end{array}$ & $\begin{array}{c}47.50 \pm \\
2.96\end{array}$ & $\begin{array}{c}57.45 \pm \\
4.44\end{array}$ & $\begin{array}{c}10.30 \pm \\
4.65\end{array}$ & $165.00 \pm 9.03$ \\
\hline
\end{tabular}

Abbreviations: G, Girls; B, Boys; SD, standard deviation

Table 2: The normal values of modified forward reach and modified lateral reach (both right and left side) among both genders between the ages of 6 to 12 years.

\begin{tabular}{|l|c|c|c|c|}
\hline \multirow{3}{*}{ Age, $\mathbf{y}$} & Gender & mFR(cm) & mLR (lt)(cm) & mLR(rt)(cm) \\
\hline \multirow{3}{*}{6} & F & $19 \pm 4.01$ & $16.80 \pm 2.87$ & $17.50 \pm 2.98$ \\
\cline { 2 - 5 } & M & $20.05 \pm 3.08$ & $18.55 \pm 1.98$ & $20.15 \pm 2.27$ \\
\cline { 2 - 5 } & F & $23.05 \pm 3.72$ & $20 \pm 3.68$ & $23.32 \pm 3.19$ \\
\hline 9 & F & $21.90 \pm 5.10$ & $22.45 \pm 4.08$ & $22.25 \pm 3.71$ \\
\cline { 2 - 5 } & M & $23.35 \pm 5.37$ & $22.80 \pm 6.37$ & $22.75 \pm 3.87$ \\
\hline 10 & F & $24.20 \pm 3.17$ & $17.85 \pm 3.20$ & $19.10 \pm 2.84$ \\
\cline { 2 - 5 } & M & $22.85 \pm 4.18$ & $20.35 \pm 6.26$ & $19.40 \pm 4.16$ \\
\hline
\end{tabular}




\begin{tabular}{|l|c|c|c|c|}
\hline & M & $22.60 \pm 7.91$ & $22.20 \pm 3.57$ & $19.95 \pm 4.32$ \\
\hline \multirow{3}{*}{11} & F & $26.80 \pm 5.84$ & $21.55 \pm 3.84$ & $19.85 \pm 3.29$ \\
\cline { 2 - 5 } & M & $26.15 \pm 4.76$ & $22.20 \pm 3.57$ & $21.80 \pm 3.79$ \\
\hline \multirow{2}{*}{12} & F & $27.45 \pm 7.46$ & $22.75 \pm 4.01$ & $21.85 \pm 4.39$ \\
\cline { 2 - 5 } & M & $24.05 \pm 6.71$ & $20.40 \pm 4.95$ & $20.85 \pm 4.71$ \\
\hline
\end{tabular}

Abbreviation: F, female; M, male; mFR, modified forward reach; $\mathrm{mLR}$, modified lateral reach

Table 3: Correlation of modified forward reach test with anthropometric measures, sit and reach distance (trunk flexibility), hamstring angle and bilateral Lateral Reach values

\begin{tabular}{|c|c|c|c|c|c|c|c|c|c|c|}
\hline Age & Gender & $\begin{array}{c}\text { Height } \\
(\mathbf{c m}) \\
(\mathbf{r})\end{array}$ & $\begin{array}{l}\text { Weight } \\
(\mathbf{K g}) \\
(\mathbf{r})\end{array}$ & $\begin{array}{c}\mathbf{B M I} \\
\left(\mathbf{K g} / \mathbf{m}^{2}\right) \\
(\mathbf{r})\end{array}$ & $\begin{array}{c}\text { Leg } \\
\text { length } \\
(\mathrm{cm}) \\
(\mathbf{r}) \\
\end{array}$ & $\begin{array}{c}\text { Trunk } \\
\text { length } \\
(\mathbf{c m}) \\
(\mathbf{r})\end{array}$ & \begin{tabular}{|c|} 
Trunk \\
flexibility $(\mathrm{cm})$ \\
$(\mathrm{r})$
\end{tabular} & $\begin{array}{c}\text { Hamstring } \\
\text { angle } \\
\text { (degrees } \\
\text { (r) } \\
\end{array}$ & $\begin{array}{c}\text { Modified Left } \\
\text { Lateral } \\
\text { reach }(\mathbf{c m}) \\
(\mathbf{r}) \\
\end{array}$ & \begin{tabular}{|c}
$\begin{array}{c}\text { Modified } \\
\text { Right Lateral } \\
\text { reach }(\mathrm{cm} \\
(\mathbf{r})\end{array}$ \\
\end{tabular} \\
\hline \multirow[t]{2}{*}{6} & $\mathrm{G}$ & 0.04 & 0.28 & 0.36 & 0.08 & 0.24 & 0.52 & 0.492 & 0.17 & $-0.17 *$ \\
\hline & B & 0.03 & $-0.01 *$ & 0.11 & $-0.12 *$ & $-0.12 *$ & 0.15 & 0.4 & 0.09 & 0.10 \\
\hline \multirow[t]{2}{*}{7} & G & 0.38 & 0.14 & $-0.30^{*}$ & 0.32 & 0.002 & 0.23 & 0.23 & 0.20 & 0.35 \\
\hline & B & 0.20 & 0.01 & 0.08 & 0.30 & 0.11 & 0.18 & 0.25 & 0.23 & 0.36 \\
\hline \multirow[t]{2}{*}{8} & $\mathrm{G}$ & 0.41 & 0.28 & $-0.02 *$ & 0.15 & 0.13 & 0.07 & $-0.10^{*}$ & 0.54 & 0.38 \\
\hline & B & -0.24 & 0.05 & 0.06 & 0.06 & 0.33 & 0.004 & 0.06 & 0.45 & 0.53 \\
\hline \multirow[t]{2}{*}{9} & $\mathrm{G}$ & 0.13 & 0.13 & 0.04 & 0.17 & 0.16 & 0.53 & 0.12 & 0.23 & 0.14 \\
\hline & B & 0.20 & $-0.11^{*}$ & 0.18 & 0.17 & 0.12 & 0.21 & $-0.05^{*}$ & 0.18 & 0.33 \\
\hline \multirow[t]{2}{*}{10} & G & -0.04 & 0.23 & 0.37 & $-0.10^{*}$ & $-0.06^{*}$ & 0.39 & 0.67 & 0.46 & 0.68 \\
\hline & $\mathrm{B}$ & -0.37 & 0.20 & 0.01 & $-0.35^{*}$ & $-0.02 *$ & 0.28 & 0.7 & 0.82 & 0.72 \\
\hline \multirow[t]{2}{*}{11} & $\mathrm{G}$ & 0.48 & 0.23 & $-0.05^{*}$ & 0.21 & 0.28 & 0.02 & 0.04 & 0.51 & 0.43 \\
\hline & B & 0.31 & 0.30 & 0.21 & $-0.39 *$ & 0.67 & 0.28 & 0.05 & 0.23 & 0.57 \\
\hline \multirow[t]{2}{*}{12} & G & $\begin{array}{l}-0.18 \\
\end{array}$ & $-0.45 *$ & $-0.58^{*}$ & $-0.08 *$ & $-0.48 *$ & $-0.19 *$ & 0.37 & 0.49 & 0.41 \\
\hline & B & 0.42 & $-0.009^{*}$ & $-0.28 *$ & 0.31 & 0.12 & $-0.12 *$ & 0.009 & 0.51 & 0.66 \\
\hline
\end{tabular}

Abbreviations: G, Girls; B, Boys; L, Left; R, Right,*, negative correlation

Abbreviations: G, Girls; B, Boys; L, Left; R, Right,*, negative correlation

Values represent Karl Pearson's Correlation Coefficient (r)

Table 4: Correlation of modified lateral reach distances with anthropometric measures, sit and reach distance (trunk flexibility) and hamstring angle

\begin{tabular}{|c|c|c|c|c|c|c|c|c|c|c|c|c|c|c|c|}
\hline \multirow[t]{2}{*}{ Age } & \multirow[t]{2}{*}{ Gender } & \multicolumn{2}{|c|}{$\begin{array}{c}\text { Height }(\mathbf{c m}) \\
(\mathbf{r})\end{array}$} & \multicolumn{2}{|c|}{$\underset{(\mathbf{r})}{\text { Weight }(K g)}$} & \multicolumn{2}{|c|}{$\begin{array}{c}\text { BMI }\left(\mathbf{k g} / \mathbf{m}^{2}\right) \\
(\mathbf{r})\end{array}$} & \multicolumn{2}{|c|}{\begin{tabular}{|c} 
Leg length $(\mathrm{cm})$ \\
$(\mathbf{r})$
\end{tabular}} & \multicolumn{2}{|c|}{$\begin{array}{c}\text { Trunk } \\
\text { length }(\mathrm{cm}) \\
(\mathrm{r})\end{array}$} & \multicolumn{2}{|c|}{$\begin{array}{c}\text { Trunk } \\
\text { flexibility } \\
(\mathbf{c m})(\mathbf{r})\end{array}$} & \multicolumn{2}{|c|}{$\begin{array}{l}\text { Hamstring } \\
\text { angle } \\
\text { (degrees) }(\mathbf{r})\end{array}$} \\
\hline & & $\mathrm{L}$ & $\mathrm{R}$ & $\mathrm{L}$ & $\mathrm{R}$ & $\mathrm{L}$ & $\mathrm{R}$ & $\mathrm{L}$ & $\mathrm{R}$ & $\mathrm{L}$ & $\mathrm{R}$ & $\mathrm{L}$ & $\mathrm{R}$ & $\mathrm{L}$ & $\mathrm{R}$ \\
\hline \multirow[t]{2}{*}{6} & $\mathrm{G}$ & 0.17 & $-0.17^{*}$ & 0.21 & 0.14 & 0.17 & 0.24 & 0.20 & 0.13 & 0.17 & $0.23 *$ & 0.15 & $0.19 *$ & 0.53 & 0.30 \\
\hline & $\mathrm{D}$ & & 028 & & & 0.40 & 00 & & & 0.07 & & 5 & 022 & $-0.24 *$ & $-0.12 *$ \\
\hline \multirow[t]{2}{*}{7} & $\bar{G}$ & -0.02 & 0.37 & $-0.13^{*}$ & 0.280 & 0.34 & \begin{tabular}{|l|}
0.003 \\
\end{tabular} & \begin{tabular}{|l|}
-0.02 \\
\end{tabular} & & 0.07 & .41 & $.04 *$ & & 0.40 & $-0.05^{*}$ \\
\hline & B & 19 & 0.14 & 15 & $-0.16^{*}$ & 0.10 & \begin{tabular}{|l|l|}
$-0.33^{*}$ \\
\end{tabular} & 0.14 & 0.0 & 0.19 & $-0.01 *$ & $-0.43^{*}$ & $-0.13^{*}$ & $-0.457 *$ & $-0.20^{*}$ \\
\hline \multirow[t]{2}{*}{8} & $G$ & & & & & & & & & 06 & & $-0.30^{*}$ & $-0.38^{*}$ & $-0.30^{*}$ & $-0.40^{*}$ \\
\hline & $\mathrm{B}$ & & $-0.37^{*}$ & & 0.0 & 0.0 & 0 & & 32 & 19 & $-0.20 *$ & $-0.18^{*}$ & 041 & -0. & 0.43 \\
\hline \multirow[t]{2}{*}{9} & $\mathrm{G}$ & 9. & 0.12 & $-0.11 *$ & $-0.15^{*}$ & $-0.06^{*}$ & \begin{tabular}{|l|}
$-0.30 *$ \\
\end{tabular} & 0.01 & & 0.23 & 0.28 & 0.05 & .182 & $-0.04 *$ & 0.007 \\
\hline & B & $-0.45^{*}$ & $-0.19^{*}$ & $-0.08 *$ & $-0.28 *$ & 0.05 & \begin{tabular}{|l|l|}
$-0.28 *$ \\
\end{tabular} & $-0.21 *$ & $-0.12 *$ & 0.02 & $-0.05 *$ & $-0.02 *$ & 0.02 & 0.15 & 0.14 \\
\hline \multirow[t]{2}{*}{10} & $\mathrm{G}$ & & & & 0. & 0. & 4 & & & 03 & & 0.50 & 4 & 0.1 & 0.37 \\
\hline & & -0 & $-0.01 *$ & & 0.03 & 0.15 & & 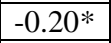 & & 04 & & & & 0. & 0.47 \\
\hline \multirow[t]{2}{*}{11} & $\mathrm{C}$ & 0.29 & 0.45 & & 0.14 & 0.05 & \begin{tabular}{|l|}
$-0.15^{*}$ \\
\end{tabular} & \begin{tabular}{|l|}
$-0.27 *$ \\
\end{tabular} & $5 *$ & 0.47 & 0.46 & 0.48 & 0.39 & $-0.14 *$ & 0.45 \\
\hline & B & 0.05 & 0.25 & 0.15 & 0.20 & 0.18 & 0.15 & 0.01 & $7 *$ & 0.22 & 0.43 & $-0.27^{*}$ & 0.24 & $-0.29 *$ & $-0.07 *$ \\
\hline \multirow[t]{2}{*}{12} & $G$ & $-0.25^{*}$ & $-0.36^{*}$ & $-0.33^{*}$ & $-0.26^{*}$ & $-0.38^{*}$ & \begin{tabular}{|l|}
$-0.20^{*}$ \\
\end{tabular} & $-0.12^{*}$ & $-0.25^{*}$ & $-0.25^{*}$ & $-0.33^{*}$ & 0.03 & $-0.21^{*}$ & 0. & 0.68 \\
\hline & B & 0.44 & 0.34 & 0.08 & 0.09 & \begin{tabular}{|l|}
$-0.16^{*}$ \\
\end{tabular} & \begin{tabular}{|l|l|}
$-0.10^{*}$ \\
\end{tabular} & 0.22 & 0.28 & 0.40 & 0.12 & 0.12 & $-0.23^{*}$ & $-0.22^{*}$ & 0.17 \\
\hline
\end{tabular}

Abbreviations: G, Girls; B, Boys; L, Left; R, Right, *, negative correlation

Values represent Karl Pearson's Correlation Coefficient ( $\mathrm{r}$ ) 
Table 5: Regression Values and coefficients of mLRT of both sides with mFRT in both genders

\begin{tabular}{|c|c|c|c|c|c|c|}
\hline \multicolumn{2}{|c|}{} & \multicolumn{2}{|c|}{ Unstandardized Coefficients } & $\begin{array}{c}\text { Standardized } \\
\text { Coefficients }\end{array}$ & \multirow{2}{*}{ t value } & \multirow{2}{*}{ p value } \\
\cline { 2 - 7 } \multicolumn{2}{|c|}{} & B & Std. Error & Beta & & \\
\hline \multirow{2}{*}{ (Constant) } & 7.280 & 1.594 & & 4.567 & 0.000 \\
\cline { 2 - 7 } & Modified Left Lateral reach(cm) & 0.401 & 0.080 & 0.322 & 5.030 & 0.000 \\
\cline { 2 - 7 } & Modified Right Lateral reach(cm) & 0.383 & 0.090 & 0.272 & 4.250 & 0.000 \\
\hline
\end{tabular}

a. Dependent Variable: Modified Forward Reach $(\mathrm{cm})$

$\mathrm{p}$ value $<0.05$ considered as statistically significant

\section{Graph 1: Correlation of mLRT on both sides in both genders}

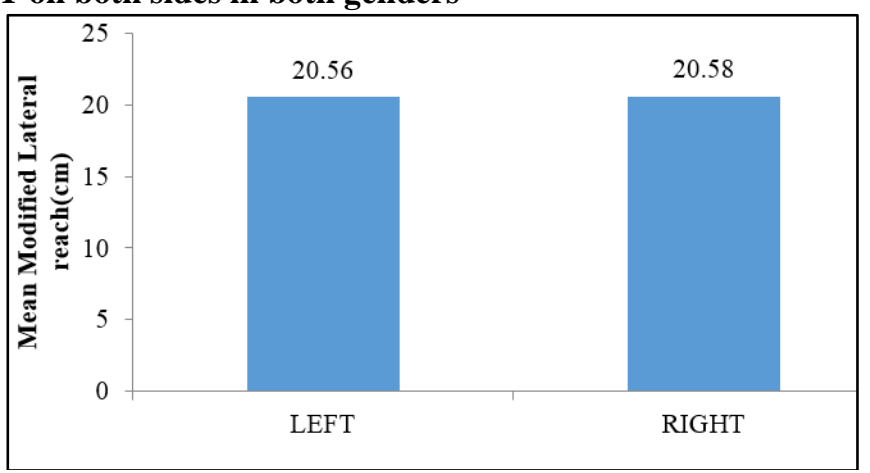

\section{Discussion}

The anthropometric measurements (height, weight, BMI) (Table 1) of the children included in the present study were in agreement with the Indian data for the respective age group. ${ }^{17,26,27}$ The values for modified forward reach and modified lateral reach in age group 6-12 years among both genders have not been established hence this study reported the reference values (Table 2).

The current study found that the modified functional reach test values were affected by the factors such as height and weight in both genders. This finding is consistent with findings of Donahoe and associates $(1996)^{20}$ where it was stated that as age advances, height and weight increases and also the functional reach. Another study by Habib and associates (1998) showed that height was the most significant factor for the FR values in Pakistani children aged 5-7 years. AA Deshmukh and associates $2011^{17}$ also found that FR values were significantly affected by height in Indian girls aged 6-12 years. Rosemary and associates $(2003)^{23}$ found that weight was the only significant factor affecting the FR values in children aged 3-5 years. This finding contradicts the studies by Thompson M (2007) and P. Singh (2013) where they did not find any significant correlation between anthropometric measures and modified functional reach test values in American and Indian adults (20-97 years) respectively.

In the present study, modified functional reach test values increases in both genders in parallel with leg length as well as trunk length. This is in accordance with the previous study by P. Singh and associates $(2013)^{6}$ where they found that mFRT and mLRT values were significantly correlated with trunk length in young Indian adults (20-39 years). Study performed on children by Tacar and colleagues $(1999)^{28}$ found that upper and lower extremity length increases in parallel to height which may explain the results of present study that, as the leg length increases the modified functional reach test values increases well.

AA Deshmukh and associates $(2011)^{17}$ also found similar results where high correlations of lengths of upper and lower extremities with FRT results were observed only in girls. In present study it is also observed that trunk flexibility had effect on mFRT values in boys. This finding is supported by Margaret $\mathrm{S}$ et al $(2000)^{29}$ who found that spinal flexibility is a contributor to functional reach values performed in standing. Balance is dependent on tissue flexibility as well as strength and there is a gender difference among children with respect to flexibility and strength. Physiologically boys are stronger than girls and at early age both boys and girls are equally flexible as stated in study by Stephen Haley and associates $(1986)^{30}$ that anterior spinal flexion measurements for both genders demonstrated no significant trend between ages of 5 and 9years. Hence in present study better trunk flexibility and strength among children might have contributed significantly for mFRT values. In this study hamstring length did not contribute to mFRT and mLRT values. This can be explained biomechanically as, in sitting position, two joint hamstring muscle is in a shortened position at knee hence reaching forward may not require complete excursion of hamstring muscles.

BMI also did not affect both reach values. The participants were of growing age and as age increased height and weight also showed an increment in their values and hence their ratio i.e. BMI remained constant. Moreover, none of the participants in the study were obese so excess adipose tissue did not contribute for limitation of movement. ${ }^{20}$ For mLRT, height, weight, leg length and trunk length were important factors in girls whereas in boys the distances were not affected by the anthropometric 
measurements. This result is in agreement with the study by AA Deshmukh and associates $(2011)^{17}$ where for lateral reach values, height was an important factor among girls. The result of previous study ${ }^{6}$ stated that trunk length was significantly correlated with lateral reach values in young Indian adults (20-39 years).

In present study, it was observed that mFRT values are more than mLRT values in both genders. This result is consistent with the study by AA Deshmukh $(2011)^{17}$ where similar results were found for functional reach tests. The reason for this could be explained as in sitting there is a larger BOS and shorter lever arm as compared to standing which allows a greater stability. Abroader BOS while reaching forward as compared to a smaller BOS while reaching lateral may be the reason for variation in reach values. While reaching forward the participants could get a visual feedback and hence may have performed better. While reaching laterally as visual feedback had been blocked, the participants depended on somatosensory system and vestibular system which is not fully developed in this age. ${ }^{1}$

Results showed that values of FR were more than $\mathrm{mFR}$ whereas LR values were lesser than $\mathrm{mLR}$ when compared with the study done by AA Deshmukh. (2011). ${ }^{17}$ During reaching forward in standing, various postural strategies are used such as hip, ankle and trunk, whereas in sitting only trunk strategy is used. In lateral reach, the BOS is broader in sitting hence children were able to reach farther as compared to the standing ${ }^{7}$. It was also found that mFRT and mLRT showed a highly significant correlation with each other i.e. mLRT values can be predicted by mFRT values and vice versa. Comparative group statistics of left and right lateral reach do not show any significant difference. Hence in the present study, only right side lateral reach distance is considered for statistical analysis. Thus from the present study it can be concluded that height and weight are the important factors for mFRT and mLRT values as, parameters like weight, height, leg length, trunk length showed direct correlations with growth.

The normal values obtained in this study can be used as baseline data for assessment of balance impairments among children aged 6-12years in India, in conditions like Cerebral palsy, Down's syndrome, Muscular dystrophies, Peripheral neuropathies, Spinal structural defects, etc. ${ }^{8-13}$ In future research, correlation of trunk strength and modified functional reach test can be evaluated. In addition to examining people with sitting balance impairments, further examination of the psychometric properties of the reaching forward and lateral in sitting position, need to be explored in larger sample size.

\section{Conclusion}

From the present study it can be concluded that, the range of normal values of mFRT are $19.53 \pm 3.57 \mathrm{~cm}$ to $26.48 \pm 5.27 \mathrm{~cm}$ and that of $\mathrm{mLRT}$ reach are $17.68 \mathrm{~cm}$ $\pm 2.59 \mathrm{~cm}$ to $22.50 \mathrm{~cm} \pm 3.75 \mathrm{~cm}$ respectively in Indian school going children of age 6-12 years. Among all the anthropometric measures, height and weight contributes significantly to both mFRT and mLRT values.

\section{Conflict of Interest: None.}

\section{References}

1. Westcott SL, Lowes LP, Richardson PK. Evaluation of postural stability in children: current theories and assessment tools. Phys Ther 1997;77:629-645.

2. Assaiante C, Amblard B. Ontogenesis of head stabilization in space during locomotion in children: influence of visual cues. Exp Brain Res 1993;93(3):499-515.

3. Riach CL, Starkes JL. Velocity of centre of pressure excursions as anindicator of postural control systems in children. Gait Posture 1994;2(3):167-172.

4. Hay L, Redon C. Feed forward versus feedback control in children and adults subjected to a postural disturbance. Exp Brain Res 1999;125(2):153-162.

5. Shumway-Cook A, Woollacott MH. Normal postural control. Motor Control Translating Research into Clinical Practice. 2001;158-186.

6. Singh P, Hujon N. Normative data of modified functional reach test in younger and middle-aged North Eastern Indian population. Arch Med Health Sci. 2013;1(2):109-114.

7. O'Sullivan SB, Schmitz TJ. Physical Rehabilitation. $5^{\text {th }}$ edition. NewDelhi, India: Jaypee Brothers. 373-399.

8. McCaslin DL, Gary PJ, Gruenwald J. The predominant forms of vertigo in children and their associated findings on balance function testing. Otolaryngol Clin North Am 2011;44(22):291307.

9. Smith RM, Emans JB. Sitting balance in spinal deformity. Spine.1992; 17(9):1103-1109

10. Lynch SM, Leahy P, Barker SP. Reliability of measurements obtained with modified functional reach test in subjects with spinal cord injury. Phys Ther 1998;78:128-133.

11. Wright MJ, Galea V, Barr RD. Proficiency of balance in children and youth who have had acute lymphoblastic leukaemia. Phys Ther 2005;85(8):782-790.

12. Patricia D ed. Cash's Textbook of Neurology for Physiotherapists. $4^{\text {th }}$ edition. New Delhi, India. 435-457,517524,552-589.

13. List of Assessment Tools Used in Paediatric Physical Therapy.Section on Paediatrics. American Physical Therapy Association.

14. Available:https://google.co.in/url?q=https://med.unc.edu/ahs/p hysical/files/school-basedptdocs/Ped\%20Assessment\%20Tools.pdf\&sa=U\&ei=pwKVZ Crll3GuATGsol4Aw\&ved=0CA8QFjAA\&usg=AFQjCNGz7q QqTyVAmhHhG0w_jfSPuzqX5A.

15. Harris SR, Gregson JL, Field D, Fife SE, Roxborough LA, Armstrong RW. Development of a clinical measure of postural control for assessment of adaptive seating in children with neuromotor disabilities. Phys Ther 1991;71:981-993.

16. Knox V. Evaluation of the sitting assessment for children with neuromotor dysfunction (SACND) as a measurement tool in cerebralpalsy: a case study. Physiother 2002;88(9):534-541.

17. Pountney TE, Cheek L, Green E, Mulcahy C, Nelham R. Content andcriterion validation of the Chailey levels of ability. Physiother 1999;85(8):410-416.

18. Deshmukh AA, Ganesan S, Tedla JL. Normal values of functional reach and lateral reach tests in Indian school children. Pediatr Phys Ther 2011;23:23-30.

19. Plisky PJ, Gorman PP, Elkins B. The reliability of an instrumented device for measuring components of the star excursion balance test. N Am J Sports Phys Ther 2009;4(2):92. 
20. Thompson M, Medley A. forward and lateral sitting functional reach in younger, middle-aged, and older adults. $J$ Geriatr Phys Ther 2007;30(2):43-47.

21. Donahoe B, Turner D, Worrell T. The use of functional reach as a measurement of balance in boys and girls without disabilities 5 to 15years. Pediatr Phys Ther 1994;6:189-193.

22. Bartlett D, Birmingham T. Validity and reliability of a pediatric reach test. Pediatr Phys Ther 2003;15:8492.doi:10.1097/01.PEP.0000067885.63909.5C

23. Habib Z, Westcott S. Assessment of anthropometric factors on balance tests in children. Pediatr Phys Ther 1998;10:101-109.

24. Norris RA, Wilder E, Norton J. Functional reach test in 3- to 5year old children without disabilities. Pediatr Phys Ther 2008;20:47-52.doi:10.1097/PEP.0b013e31815ce63f.

25. Marwah RK, Tandon N, Ganie MA, Kanwar R, Shivprasad C, Sabharwal A, et al. Nationwide reference data for height, weight and body mass index of Indian schoolchildren. Ntl Med J India 2011;24(5):269-277.
26. Chakravarti D. Indian anthropometric dimensions for ergonomic design practice. NID 1997.

27. Katz K, Rosenthal A, Yosipovitch Z. Normal ranges of popliteal angle in children. J Pediatr Orthop 1992;12(2):229231.

28. Ghai OP ed., Gupta P ed., Paul VK. Essential Pediatrics. 6th edition. Delhi: CBS Publisher and distributor: 2005.

29. Tacar O, Dogruyol S, Hatipoglu ES. Lower and upper limb length of urban and rural 7-11 years old Turkish school children. Anthropol Anz 1999;57:269-276.

30. Schenkman M, Morey M, Kuchibhatla M. Spinal flexibility and balance control among community-dwelling adults with and without parkinson's disease. J Gerontol A Bio Sci Med Sci 2000; 55(8):M441-M445. doi:10.1093/Gerona/55.8.M441.

31. Haley SM, Tada WL, Carmichael EM. Spinal mobility in young children a normative study. Phys Ther 1986;66:16971703. 